\title{
Traqueobroncopatía osteocondroplástica: Reporte de un caso y revisión de la literatura
}

\author{
Tracheobronchopathy osteochondroplastica: A case report and literature review
}

\author{
Carlos Celedón L¹, José de Grazia $\mathrm{K}^{2}$.
}

\begin{abstract}
RESUMEN
La traqueobroncopatía osteocondroplástica es una enfermedad benigna poco frecuente, de etiología incierta, caracterizada por múltiples nódulos submucosos cartilaginosos u óseos en tráquea, bronquios principales y menos frecuentemente, región subglótica y laringe. La presentación clínica de la enfermedad varía desde pacientes asintomáticos, hasta enfermedad severa con obstrucción de la vía aérea. El gold standard para el diagnóstico es la broncoscopía, donde característicamente se visualiza la presencia de múltiples nódulos submucosos que se proyectan hacia el lumen de la vía aérea. La biopsia no es requisito para el diagnóstico. El tratamiento de la enfermedad está dirigido habitualmente al manejo sintomático. En pacientes con severa obstrucción de la vía aérea se han realizado terapias broncoscópicas como ablación con láser, crioterapia o divulsión mecánica de las lesiones. También se ha intentado la resolución quirúrgica, siendo generalmente inefectiva. Presentamos el caso clínico de un paciente evaluado en nuestro servicio, en quien se realizó diagnóstico de traqueobroncopatía osteocondroplástica con compromiso tráqueo-bronquial y subglótico.
\end{abstract}

Palabras clave: Traqueobroncopatía osteocondroplástica, estenosis subglótica

\begin{abstract}
Tracheobronchopathy osteochondroplastica is a rare benign disease of uncertain etiology, characterized by multiple cartilaginous or bony submucosal nodules in trachea, bronchi and, less frequently, subglottic region and larynx. The clinical presentation of the disease varies from asymptomatic patients to severe disease with airway obstruction. The gold standard for diagnosis is bronchoscopy, which typically displays the presence of multiple submucosal nodules that project into the lumen of the airway. Biopsy is not required for diagnosis. The treatment of the disease is usually symptomatic management. In patients with severe airway obstruction bronchoscopic therapies such as laser ablation, cryotherapy or mechanical removal have been performed. Surgical resolution has also been attempted, being generally ineffective. We
\end{abstract}

1 Médico Otorrinolaringólogo. Hospital Clínico Universidad de Chile.

2 Interno de Medicina. Facultad de Medicina, Universidad de Chile. 
report the case of a patient evaluated in our department, in whom the diagnosis of tracheobronchopathy osteochondroplastica with tracheobronchial and subglottic compromise was made.

Key words: Tracheobronchopathy osteochondroplastica, subglottic stenosis.

\section{INTRODUCCIÓN}

La traqueobroncopatía osteocondroplástica (TBOC) es una enfermedad benigna poco frecuente, caracterizada por el desarrollo de múltiples nódulos submucosos cartilaginosos u óseos en las paredes de tráquea, bronquios principales y, menos frecuentemente, región subglótica y laringe, que puede llevar al estrechamiento del lumen de la vía aérea. Los nódulos comprometen las paredes anterior y lateral, respetando la pared posterior membranosa, debido a la ausencia de cartílago en esta región de la vía aérea ${ }^{1-3}$.

La etiología de la enfermedad es incierta ${ }^{1,2}$, al igual que su asociación con otras patologías ${ }^{1-5} 0$ condiciones predisponentes ${ }^{1,2}$. Presenta una mayor frecuencia en hombres, siendo diagnosticada mayormente entre la cuarta y sexta década de vida $^{1,2,6,8}$.

La presentación clínica de la enfermedad varía desde el diagnóstico incidental en pacientes asintomáticos, hasta la enfermedad severa con obstrucción importante de la vía aérea ${ }^{1,2}$. Los síntomas más frecuentes son disnea de esfuerzos, tos crónica, producción crónica de esputo, hemoptisis recurrente y dolor torácico $0^{1,2,3,6}$, siendo usualmente mal diagnosticada como asma bronquial ${ }^{1-3}$, enfermedad pulmonar obstructiva crónica ${ }^{1,2} 0$ incluso insuficiencia cardiaca congestiva ${ }^{7}$. Al examen físico es posible hallar sibilancias y/o estridor. Dentro de sus complicaciones se cuentan las infecciones respiratorias a repetición, bronquiectasias y atelectasias ${ }^{1,2,8}$, no existiendo evidencia suficiente que la asocie con progresión a malignidad ${ }^{2,9}$.

Los estudios de imágenes desempeñan un rol en sugerir el diagnóstico de TBOC y en realizar seguimiento de los pacientes ya diagnosticados ${ }^{1,2}$. El gold standard para el diagnóstico de TBOC es la broncoscopía, donde característicamente se visualiza la presencia de múltiples nódulos submucosos de 1 a $6 \mathrm{~mm}$ de diámetro, aislados 0 confluentes, que se proyectan hacia el lumen de la vía aérea, confiriéndole un aspecto de jardín de rocas $^{1,2}$. La biopsia no es requisito para el diagnóstico, siendo en muchas ocasiones imposible de obtener mediante broncoscopía, debido a la consistencia extremadamente dura de las lesiones ${ }^{1}$. Los hallazgos histológicos característicos corresponden a presencia de cartílago a nivel submucoso (continuo 0 aislado de los anillos cartilaginosos de la vía aérea), osificación y calcificación submucosa, médula ósea hematopoyética entre las áreas osificadas y, en ocasiones, metaplasia escamosa del epitelio respiratorio ${ }^{2}$.

El tratamiento de la enfermedad está dirigido habitualmente al manejo de los síntomas con broncodilatadores y corticoides inhalados, y al manejo de potenciales infecciones respiratorias mediante antibioticoterapia ${ }^{1,2}$. En pacientes con severa obstrucción de la vía aérea se han realizado terapias broncoscópicas como ablación con láser, crioterapia 0 divulsión mecánica de las lesiones ${ }^{1,2,4,8,10,11}$. También se ha intentado la resolución quirúrgica ${ }^{1,2,12,13}$, siendo no efectiva en muchas ocasiones, debido al compromiso difuso y multisegmentario de la vía aérea. Existen reportes escasos de manejo mediante radioterapia ${ }^{1,2}$. En ocasiones son necesarias medidas extraordinarias como la colocación de un stent traqueal en caso de obstrucción severa de la vía aérea, o la realización de traqueostomía en pacientes con compromiso de tráquea proximal, región subglótica 0 laringe $\mathrm{e}^{1,2}$. Debido a la baja prevalencia de la enfermedad, no se dispone de guías clínicas estandarizadas para su manejo $0^{1-3}$.

A continuación se presenta el caso clínico de un paciente evaluado en el Servicio de Otorrinolaringología del Hospital Clínico de la Universidad de Chile con diagnóstico de TBOC con compromiso tráqueo-bronquial y subglótico.

\section{CASO CLÍNICO}

Paciente de 66 años de edad, con antecedente de diabetes mellitus tipo 2 en tratamiento con 
hipoglicemiantes orales. Presenta cuadro de una semana de evolución caracterizado por compromiso del estado general, disnea de esfuerzos, náuseas, vómitos y un episodio de convulsiones tónico-clónicas generalizadas. Es evaluado por médico en forma domiciliaria, donde se constata compromiso de conciencia con puntaje Glasgow 7 y se intenta intubación orotraqueal que resulta infructuosa. Es trasladado a nuestro hospital, donde se realizan exámenes de laboratorio, destacando glicemia $500 \mathrm{mg} / \mathrm{dl}$, cetonemia (+) y $\mathrm{pH}$ plasmático 7,1. Se realiza diagnóstico de cetoacidosis diabética, siendo tratado con insulina, aporte de volumen parenteral y bicarbonato. El paciente evoluciona de forma favorable, con regresión del cuadro clínico al cabo de 72 horas, pero con persistencia de la disnea de esfuerzos y con presencia de estridor laríngeo espiratorio.

Se realiza radiografía de tórax, que evidencia una importante disminución del calibre de la tráquea y bronquios principales, cuyos contornos se observan además irregulares. Se decide complementar el estudio con tomografía computarizada de tórax y cuello. A nivel torácico se observa el

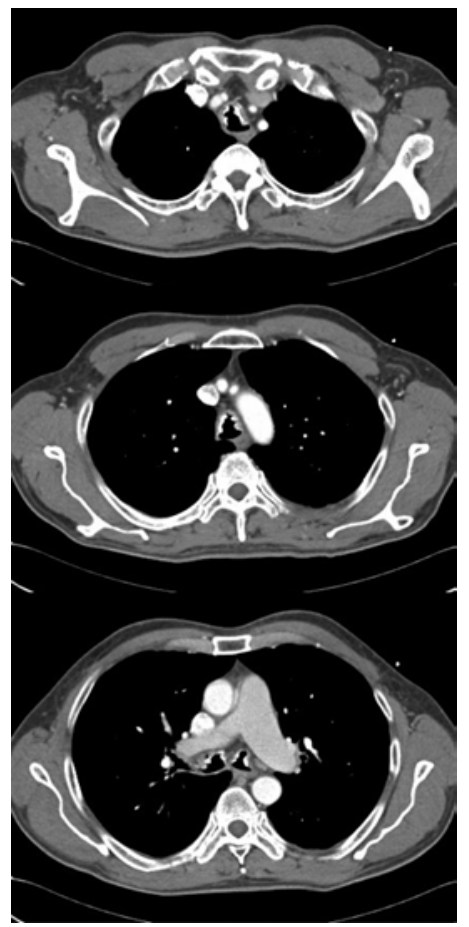

Figura 1. Tomografía computarizada de tórax, cortes transversales, en paciente con diagnóstico de TBOC. lumen de la tráquea y bronquios principales discretamente disminuido por extenso compromiso parietal, caracterizado por engrosamientos nodulares de densidad cálcica que respetan la pared posterior. No se observan alteraciones relevantes del parénquima pulmonar ni del mediastino (Figura 1). En cuello destaca la presencia de calcificaciones que se ubican en el aspecto inferior de las cuerdas vocales, extendiéndose hacia la región subglótica, donde determinan una zona de estenosis severa (Figura 2).

Debido a los hallazgos radiológicos anteriormente descritos se decide realizar videobroncoscopía, donde se aprecia mucosa nasal atrófica con descarga nasal posterior purulenta, además, se aprecian cuerdas vocales con movilidad limitada y tumoraciones óseas que comprometen difusamente la región subglótica, lo cual determina una estenosis de aproximadamente $80 \%$ de la luz. No se logra flanquear dicha estenosis, pero se logra observar la tráquea comprometida difusamente por tumoraciones óseas (Figura 3). Se intenta tomar biopsia de las lesiones, resultando infructuoso. Con los hallazgos anteriores se plantean los diagnósticos de rinitis crónica atrófica y TBOC. Se complementa el estudio con una evaluación por otorrinolaringología, para evaluación de la función laríngea. Mediante nasofibroscopía se evidencian cuerdas vocales móviles y simétricas, con estenosis severa a nivel subglótico, con dinámica laríngea adecuada.

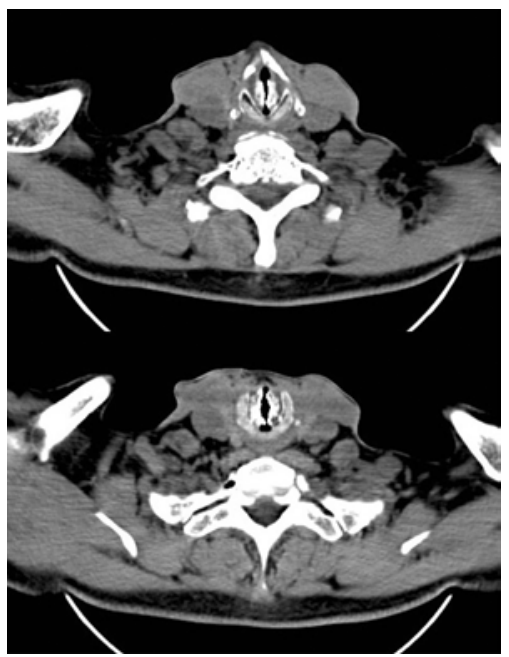

Figura 2. Tomografía computarizada de cuello, cortes transversales, en paciente con diagnóstico de TBOC. 


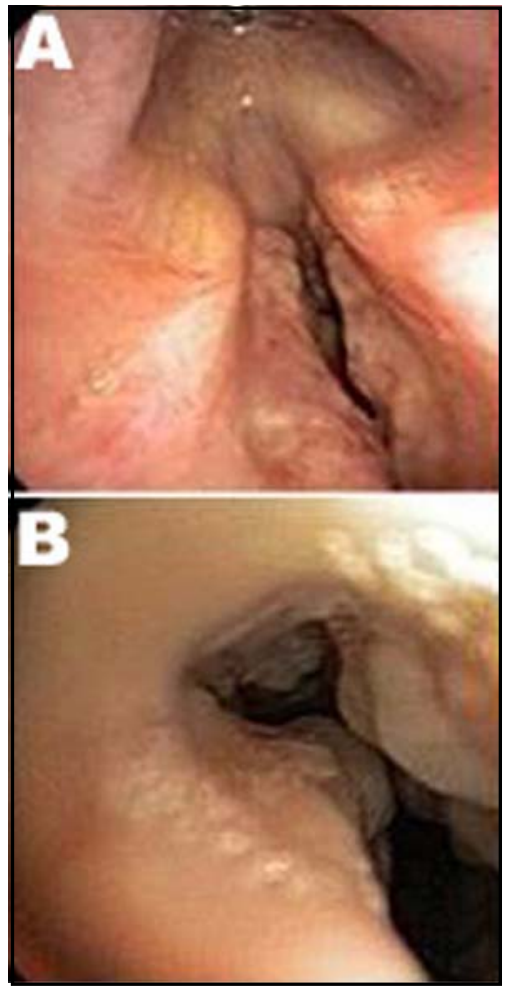

Figura 3. Videobroncoscopía en paciente con diagnóstico de TBOC. (A) cuerdas vocales; (B) visión de la tráquea.

Los hallazgos clínicos, radiológicos y endoscópicos permiten realizar el diagnóstico de TBOC con compromiso traqueobronquial y subglótico. Debido a la sintomatología leve del paciente y al carácter benigno de la enfermedad, se decide tener una conducta expectante y se da de alta al paciente.

Cuatro meses después al paciente se le realiza traqueostomía en centro hospitalario externo. Acude a control a nuestro hospital 3 meses después de realizada la traqueostomía, con persistencia de disnea de esfuerzos. Se realiza nasofibroscopía de control, por vía nasal y a través de la traqueostomía. En las visiones obtenidas de la tráquea se observa la presencia de múltiples nódulos submucosos de distribución difusa, con aspecto en jardín de rocas, los cuales respetan la pared posterior membranosa y determinan una importante disminución del lumen (Figura 4). La severidad de la obstrucción es similar a lo observado en la videobroncoscopía realizada 7 meses antes.

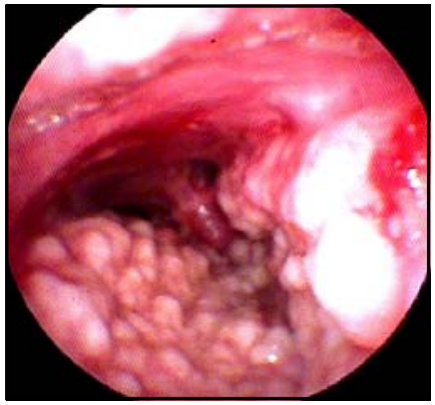

Figura 4. Nasofibroscopía en paciente con diagnóstico de TBOC, visión de tráquea.

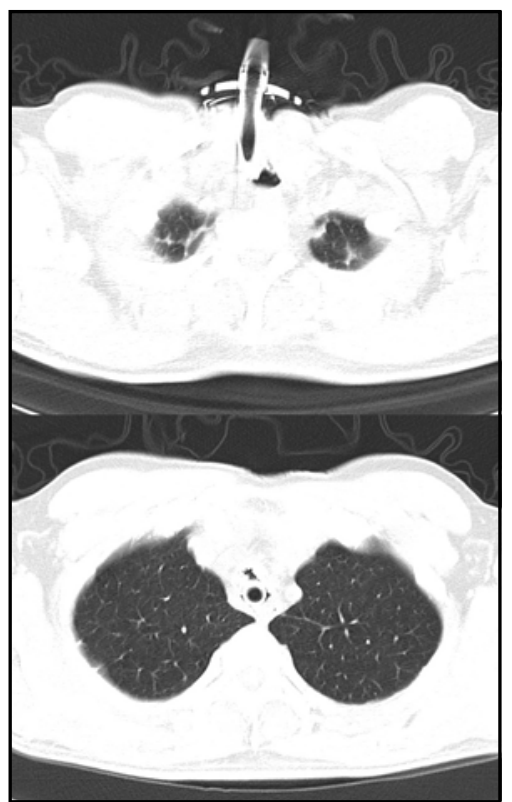

Figura 5. Tomografía computarizada de tórax, cortes transversales, en paciente con diagnóstico de TBOC con instalación de tubo T de Montgomery.

Por motivos técnicos y previsionales, el paciente es derivado a centro hospitalario externo, donde es evaluado por equipo de cirugía de tórax y se decide la instalación de tubo T de Montgomery. Posterior a este procedimiento el paciente evoluciona favorablemente, con disminución de la disnea. Acude a control médico en nuestro hospital 14 días después de instalado el tubo $T$ de Montgomery, observándose al paciente asintomático, con el tubo permeable, funcionando de manera adecuada (Figura 5). 


\section{DISCUSIÓN}

\section{Diagnóstico de TBOC}

La presentación clínica de la TBOC varía desde el diagnóstico incidental en pacientes asintomáticos, hasta la enfermedad con obstrucción severa de la vía aérea ${ }^{1,2}$. En el caso de nuestro paciente, el motivo de ingreso lo constituyó el cuadro de cetoacidosis diabética. Tras la resolución de este cuadro el paciente presentó síntomas respiratorios, que muy probablemente presentaba anteriormente, pero eran leves y habían pasado desapercibidos. Debido a estos síntomas se realizaron estudios de imágenes, en los cuales surgió la sospecha diagnóstica de TBOC. Posteriormente a esto, se amplió el estudio con broncoscopía. Este examen constituye el gold standard para el diagnóstico de TBOC $^{1,2}$. En nuestro paciente fue posible visualizar la presencia de múltiples nódulos proyectados hacia el lumen de la vía aérea, con aspecto de jardín de rocas, lo cual confirmó el diagnóstico. En los estudios broncoscópicos se intentó realizar biopsia, pero, tal como describe la literatura internacional ${ }^{1}$, las muestras fueron imposibles de obtener mediante broncoscopía, debido a la consistencia extremadamente dura de las lesiones.

\section{Compromiso laríngeo y subglótico en TBOC}

La TBOC habitualmente compromete las paredes de tráquea, bronquios principales y, menos frecuentemente, región subglótica y laringe ${ }^{1-3}$. La incidencia de la enfermedad es baja, habiéndose reportado cifras de $0,11 \%$ en pacientes sometidos a broncoscopía 6 . El compromiso laríngeo y subglótico se describe hasta en $40 \%$ de los pacientes con TBOC 8 . En el caso de nuestro paciente, el compromiso subglótico se hizo evidente tanto en la tomografía computarizada de cuello, como en la videobroncoscopía y la nasofibroscopía. Este último examen, realizado por el equipo de otorrinolaringología, demostró que, a pesar del extenso compromiso subglótico (80\%-90\% del lumen), las cuerdas vocales persistían móviles y simétricas, con dinámica laríngea adecuada.

\section{Asociación de TBOC con otras enfermedades}

Es incierta la asociación de TBOC con otras patologías tales como amiloidosis, silicosis o infección por mycobacterias ${ }^{1-5}$. No existe tampoco evidencia suficiente que asocie esta enfermedad con progresión a malignidad ${ }^{2,9}$. Resulta interesante el hecho de que nuestro paciente presentaba concomitantemente el diagnóstico de rinitis crónica atrófica. Algunos reportes extranjeros han descrito la asociación de TBOC con ozena ${ }^{4}$. La ozena se define como rinitis atrófica con descarga mucopurulenta, mucosa nasal costrosa y fetor. Un estudio finlandés describe que 6 de 18 pacientes con diagnóstico de TBOC presentaron en forma concomitante ozena 0 sinusitis maxilar recurrente ${ }^{3}$. A la luz de estos datos, no resulta extraña la asociación entre TBOC y rinitis crónica atrófica encontrada en nuestro paciente.

\section{Tratamiento de TBOC}

El tratamiento de los casos leves de TBOC incluye el manejo de los síntomas con broncodilatadores y corticoides inhalados y el manejo de potenciales infecciones respiratorias mediante antibioticoterapia ${ }^{1,2}$. En pacientes con obstrucción severa de la vía aérea se han realizado terapias broncoscópicas ${ }^{1,2,4,8,10,11} \mathrm{e}$ incluso se ha intentado la resolución quirúrgica ${ }^{1,2,12,13}$. No se dispone de guías clínicas estandarizadas para el manejo de esta enfermedad ${ }^{1-3}$. En el caso de nuestro paciente, debido al compromiso difuso y multisegmentario de la vía aérea, la opción broncoscópica y quirúrgica resultaban inviables. En primera instancia se realizó traqueostomía y luego se instaló un tubo T de Montgomery, herramienta de gran utilidad en casos de estenosis traqueales extensas que no admiten corrección quirúrgica, que presta utilidad como traqueostomía y elemento de sostén a la $v e z^{14}$. Además, es un material inerte a la reacción de los tejidos, de tal forma que puede ser usado por largo tiempo ${ }^{14}$.

\section{CONCLUSIONES}

La TBOC es una enfermedad de baja frecuencia que afecta principalmente las paredes de tráquea y bronquios principales. La laringe y región subglótica sólo se comprometen en el $40 \%$ de estos pacientes, pudiendo incluso producir una estenosis subglótica que requiera traqueostomía. 
La presentación clínica de la enfermedad varía desde el diagnóstico incidental en pacientes asintomáticos, hasta la enfermedad severa con obstrucción importante de la vía aérea. Frente al diagnóstico diferencial de disnea y tos crónica de origen no precisado se debe tener presente la TBOC.

\section{BIBLIOGRAFÍA}

1. Prakash UB. Tracheobronchopathia osteochondroplastica. Semin Respir Crit Care Med 2002; 23:167-75.

2. Abu-HiJleh M, Lee D, Braman SS. Tracheobronchopathia osteochondroplastica: a rare large airway disorder. Lung 2008; 186: 353-9.

3. Vilkman S, Keistinen T. Tracheobronchopathia osteochondroplastica. Report of a young man with severe disease and retrospective review of 18 cases. Respiration 1995; 62(3): 151-4.

4. Harma RA, SuURKari S. Tracheopathia chondroosteoplastica. A clinical study of thirty cases. Acta Otolaryngol 1977; 84(1-2): 118-23.

5. Alroy GG, Lichtig C, Kaftori JK. Tracheobronchopathia osteoplastica: end stage of primary lung amyloidosis? Chest 1972; 61(5): 465-8.

6. Jabbardarjani HR, Radpey B, Kharabian S, MasjedI MR. Tracheobronchopathia osteochondroplas- tica: presentation of ten cases and review of the literature. Lung 2008; 186(5): 293-7.

7. Hussain K, GILbeRT S. Tracheopathia osteocondroplastica. Clin Med Res 2003; 1(3): 239-42.

8. Nienhuis DM, Prakash UB, Edell ES. Tracheobronchopathia osteochondroplastica. Ann Otol Rhinol Laryngol 1990; 99(9 Pt 1): 689-94.

9. Karlikaya C, Yüksel M, Kilıçli S, Candan L. Tracheobronchopathia osteochondroplastica. Respirology 2000; 5(4): 377-80.

10. Birzgalis A, Farrington W, O’Keefe L, Shaw J. Localized tracheopathia osteoplastica of the subglotis. J Laryngol Otol 1993; 107: 352-3.

11. Gleich L, Rebeiz E, Pankratov M, Shapshay $S$. The holmium: YAG lasser assisted otolaryngologic procedures. Arch Otolaryngol Head Neck Surg 1995; 121: 1162-6.

12. GRILLO HC, WRIGHT CD. Airway obstruction owing to tracheopathia osteoplastica: treatment by linear tracheoplasty. Ann Thorac Surg 2005; 79: 1676-81.

13. Kutlu CA, Yeginzu A, Ozalp T, Baran R. Modified slide tracheoplasty for the management of tracheobroncopathia osteocondroplastica. Eur J Cardiothorac Surg 2002; 21: 140-2.

14. IÑíguez R, Der $C$, Nazar G, Iñíguez R. Resultados del uso de tubo de Montgomery. Rev Otorrinolaringol Cir Cab-Cuello 2000; 60: 17580. 